PROCEEDINGS OF THE

AMERICAN MATHEMATICAL SOCIETY

Volume 127, Number 8, Pages 2303-2314

S 0002-9939(99)04802-9

Article electronically published on April 8, 1999

\title{
INTEGRAL REPRESENTATION FORMULA FOR GENERALIZED NORMAL DERIVATIONS
}

\author{
DANKO R. JOCIĆ
}

(Communicated by Palle E. T. Jorgensen)

\begin{abstract}
For generalized normal derivations, acting on the space of all bounded Hilbert space operators, the following integral representation formulas hold:

(1) $f(A) X-X f(B)=\int_{\sigma(A)} \int_{\sigma(B)} \frac{f(z)-f(w)}{z-w} E(d z)(A X-X B) F(d w)$,

and

$$
\|f(A) X-X f(B)\|_{2}^{2}
$$$$
=\int_{\sigma(A)} \int_{\sigma(B)}\left|\frac{f(z)-f(w)}{z-w}\right|^{2}\|E(d z)(A X-X B) F(d w)\|_{2}^{2},
$$

whenever $A X-X B$ is a Hilbert-Schmidt class operator and $f$ is a Lipschitz class function on $\sigma(A) \cup \sigma(B)$. Applying this formula, we extend the FugledePutnam theorem concerning commutativity modulo Hilbert-Schmidt class, as well as trace inequalities for covariance matrices of Muir and Wong. Some new monotone matrix functions and norm inequalities are also derived.
\end{abstract}

\section{INTRODUCTION}

In his paper [Ki] Kittaneh proved the following theorem for bounded Hilbert space operators:

Theorem 1.1. Let $A$ and $B$ be normal bounded operators such that $A X-X B$ belongs to the Hilbert-Schmidt class for some bounded operator $X$, and let $f$ be a Lipschitz function defined on $\sigma(A) \cup \sigma(B)$. Then $f(A) X-X f(B)$ also belongs to the Hilbert-Schmidt class; moreover,

$$
\|f(A) X-X f(B)\|_{2} \leq L\|A X-X B\|_{2},
$$

where $L$ stands for the Lipschitz constant associated to $f$.

The proof presented there is based on a famous Voiculescu theorem representing every normal operator as a sum of one diagonal and one Hilbert-Schmidt class operator which has the arbitrary small Hilbert-Schmidt norm (see [Vo]). If $A=B$ is self-adjoint, then (3) holds for arbitrary closed derivations, as shown recently in

Received by the editors January 2, 1997 and, in revised form, October 28, 1997.

1991 Mathematics Subject Classification. Primary 47A13, 47B10, 47B15, 47B47, 47B49; Secondary 47A30, 47A60.

Key words and phrases. Double operator integrals, unitarily invariant norms, Ky-Fan dominance property.

(C)1999 American Mathematical Society 
[Sh]. The aim of this paper is to show that this one, just like several other results, including Muir and Wong trace inequality

$$
\operatorname{tr}(A-B)\left(A^{-1}-B^{-1}\right) \leq 0
$$

for covariance matrices $A$ and $B$, can be seen as a consequence of the above integral representations (1) and (2). As known, some double integral formulas similar to (1) have already been discussed in [BS66], [BS68] and [BS69], but only under different additional conditions: for a pair of unitary or self-adjoint operators $A$ and $B, X=I$, the identy operator and $f^{\prime}$ belonging to $\operatorname{Lip}(\alpha)$ class for some $\alpha>0$ ([BS66] Th.10), or for a pair of normal operators $A$ and $B$, a Hilbert-Schmidt class operator $X$ and for a Lipschitz function $f$ ([BS66] Th.1, [BS68] Th.1 and their preliminaries). For matrices, a formula of this type has been treated in [HJ]. The basic intention of this paper is to extend formulas (1) and (2) to all bounded operators $X$ having Hilbert-Schmidt class generalized normal derivation (g.n.d.) $A X-X B$, and finally, to present some typical applications.

Thoughout this paper $\mathfrak{B}(\mathcal{H})$ and $\mathfrak{C}_{\infty}(\mathcal{H})$ will stand respectively for spaces of all bounded and all compact linear operators acting on a separable, infinite-dimensional complex Hilbert space $\mathcal{H}$. For an $X \in \mathfrak{B}(\mathcal{H})$ let $\sigma(X)$ and $\|X\|$ denote its spectrum and norm respectively. Following [GK], for an arbitrary $X \in \mathcal{C}_{\infty}(\mathcal{H})$, let $s_{1}(X) \geq s_{2}(X) \geq \cdots$ denote the singular values of $X$, i.e., the eigenvalues of $|X|=\sqrt{X^{*} X}$, arranged in a non-increasing order, with their multiplicities counted. Each "symmetric gauge function" $\Phi$ on sequences gives rise to a unitarily invariant (u.i.) norm on operators defined by $\|A\|_{\Phi}=\Phi\left(\left\{s_{n}(A)\right\}\right)$. We will denote by the symbol $\|\cdot\|$ any such norm, which is defined on a natural subclass $\mathcal{C}_{\|\cdot\|}$ of $\boldsymbol{C}_{\infty}$ called the norm ideal associated with the norm $\|\cdot\|$ and satisfies the invariance property

$$
\|U X V\|=\|X\| \quad \text { for all } X \in \mathbf{C}_{\|\cdot\|} \text { and for all unitary } U \text { and } V .
$$

Especially well known among unitarily invariant norms are the Schatten $p$ norms defined as $\|X\|_{p}=\sqrt[p]{\sum_{i=1}^{\infty} s_{i}^{p}(X)}$ if $1 \leq p<\infty$, and $\|X\|_{\infty}=s_{1}(X)$. They represent the norm on the associated ideal $\mathfrak{C}_{p}(\mathcal{H})$ known as the Schatten $p$-class. For a complete account of the theory of norm ideals, the reader is refered to $[\mathrm{GK}],[\mathrm{GGK}]$ and $[\mathrm{Si}]$. In this paper we will be particularly interested in operators belonging to $\mathfrak{C}_{2}(\mathcal{H})$, which is also known as the Hilbert-Schmidt class, and which represents a Hilbert space itself when equipped with the inner product $\langle X, Y\rangle=\operatorname{tr}\left(X Y^{*}\right)$. Norm $\|\cdot\|_{2}$ is lower semicontinuous, i.e., $\left\|w-\lim _{n \longrightarrow \infty} X_{n}\right\|_{2} \leq$ $\liminf _{n \longrightarrow \infty}\left\|X_{n}\right\|_{2}$, as follows from the well known representation formula $\|X\|_{2}=$ $\sup \left\{\frac{|\operatorname{tr}(X Y)|}{\|Y\|_{2}}: Y\right.$ is finite dimensional $\}$.

The Ky-Fan norms defined as $\|X\|_{k}=\sum_{i=1}^{k} s_{i}(X)$ for $k=1,2, \ldots$, represent another interesting family of unitarily invariant norms. The property saying that for all $X \in \mathcal{C}_{\infty}$ and $Y \in \mathcal{C}_{\|\cdot\| \|}$ with $\|X\|_{k} \leq\|Y\|_{k}$ for all $k \geq 1$ we have $X \in \mathfrak{C}_{\|\cdot\|}$ with $\|X\| \leq\|Y\|$ is known as the Ky-Fan dominance property.

Throughout this paper, for a given $A$ and $B$ in $\mathfrak{B}(\mathcal{H})$, a symbol $\mathcal{A}$ will be reserved for the left multiplication by $A$ and $\mathcal{B}$ for the right multiplication by $B$ on $\mathcal{C}_{2}(\mathcal{H})$. Also, we will reserve $A$ and $B$ to denote normal bounded operators on $\mathcal{H}$ (selfadjointness not excluded), while $E$ and $F$ will stand respectively, for their associated spectral measures. As a standard spectral theory fact says, those measures are concentrated on spectrum of their associated operators, i.e., $\operatorname{supp}(E)=\sigma(A)$ and $\operatorname{supp}(F)=\sigma(B)$. For such $A$ and $B$ the mapping $\Delta: \mathfrak{B}(\mathcal{H}) \longrightarrow \mathfrak{B}(\mathcal{H})$ given by 
$\Delta(X)=A X-X B$ is called a generalized normal derivation, and, as usual, the same name will also be used for $A X-X B$ itself. Special aspects of normal derivations were treated in $[\mathrm{Fi}]$ and $[\mathrm{R} 56]$.

\section{Double OPERATOR INTEGRALS}

For analytic (in each variable) functions, defined in a neighbourhood of the Cartesian product of the spectrum of two arbitrary bounded Hilbert space operators, the corresponding functional calculus on $\mathfrak{B}(\mathcal{H})$, sometimes under different names, was considered in detail by many authors (see $[\mathrm{CF}]$ or $[\mathrm{DK}]$ for details). For essentially bounded Borel functions defined on the Cartesian product of the spectrum of two normal operators, the corresponding functional calculus could not, in general, be defined on the whole $\mathfrak{B}(\mathcal{H})$ (see [Fa] for a counterexample), but merely on $\mathfrak{C}_{2}(\mathcal{H})$, which is, therefore, a natural framework for introducing double operator integrals. For their presentation, we will use a slightly different approach than this one given in [BS66] and [BS68], where one can also find more details about integration in other Schatten classes. We start with the following statement from [J93], which is a starting point for an introduction of double operator integrals.

Theorem 2.1. For given $X$ and $Y$ in $\mathfrak{C}_{2}(\mathcal{H})$, the mapping $\mu_{X, Y}$ defined on (Borel) rectangles in $\mathbb{C}^{2}$ by $\mu(\gamma \times \delta)=\operatorname{tr}\left(E(\gamma) X F(\delta) Y^{*}\right)$ has a unique extension to a complex Borel measure on $\mathbb{C}^{2}$, such that $\left|\mu_{X, Y}\right|\left(\mathbb{C}^{2}\right) \leq\|X\|_{2}\|Y\|_{2}$.

For a more prospective insight, here we present some details of the proof. So, whenever $X=Y$, the corresponding mapping $\mu_{X}=\mu_{X, X}$ is positive, finite and $\sigma$-additive on rectangles, and therefore, it can effectively be extended to at least all Borel sets in $\mathbb{C}^{2}$ by a standard Caratheodory extension procedure. For arbitrary $X$ and $Y$ in $\mathcal{C}_{2}(\mathcal{H})$, let us note that $\mu_{X, Y}=\frac{1}{4}\left(\mu_{X+Y}-\mu_{X-Y}+\imath \mu_{X+\imath Y}-\imath \mu_{X-\imath Y}\right)$ holds for rectangles, and therefore, $\mu_{X, Y}$ is a restriction of the right hand side to Borel sets in $\mathbb{C}^{2}$. Next, we see that for its total variation $\left|\mu_{X, Y}\right|$ we have an estimate

$$
\left|\mu_{X, Y}\right|(\partial)^{2} \leq \mu_{X}(\partial) \mu_{Y}(\partial)
$$

for all Borel sets $\partial \subset \mathbb{C}^{2}$. First, following the lines of the proof of Theorem 2.1 in [J93], we see that

$$
\left|\mu_{X, Y}(\partial)\right|^{2} \leq \mu_{X}(\partial) \mu_{Y}(\partial),
$$

whenever $\partial \in \Sigma^{1}$, i.e., whenever $\partial$ is representable as at most a countable union of mutually disjoint rectangles $\gamma_{n} \times \delta_{n}$. According to the properties of outer measure, for an arbitrary Borel measurable $\partial$ and a given $\epsilon>0$, there is a $\Sigma^{1}$ set $\partial_{\epsilon} \supset \partial$ such that $0 \leq \mu_{X+\imath^{k} Y}\left(\partial_{\epsilon}\right)-\mu_{X+\imath^{k} Y}(\partial)<\epsilon$, for $k=0,1,2,3$. Then $0 \leq \mu_{X}\left(\partial_{\epsilon}\right)-\mu_{X}(\partial)<$ $\epsilon$ and $0 \leq \mu_{Y}\left(\partial_{\epsilon}\right)-\mu_{Y}(\partial)<\epsilon$ as well, because $\mu_{X+Y}+\mu_{X-Y}=2 \mu_{X}+2 \mu_{Y}$. Therefore,

$$
\left|\mu_{X, Y}(\partial)\right| \leq \epsilon+\left|\mu_{X, Y}\left(\partial_{\epsilon}\right)\right| \leq \epsilon+\sqrt{\mu_{X}\left(\partial_{\epsilon}\right) \mu_{Y}\left(\partial_{\epsilon}\right)} \leq \sqrt{\left(2 \epsilon+\mu_{X}(\partial)\right)\left(2 \epsilon+\mu_{Y}(\partial)\right)},
$$

due to (5). Letting $\epsilon \longrightarrow 0$, we see that (5) holds for all Borel sets $\partial$. Finally, for all at most countable partitions $\left\{\partial_{n}\right\}$ of $\partial$, we may conclude (4) because

$$
\begin{aligned}
& \sum_{n}\left|\mu_{X, Y}\left(\partial_{n}\right)\right| \leq \sum_{n} \sqrt{\mu_{X}\left(\partial_{n}\right) \mu_{Y}\left(\partial_{n}\right)} \\
& \leq \sqrt{\sum_{n} \mu_{X}\left(\partial_{n}\right) \sum_{n} \mu_{Y}\left(\partial_{n}\right)}=\sqrt{\mu_{X}\left(\bigcup_{n} \partial_{n}\right) \mu_{Y}\left(\bigcup_{n} \partial_{n}\right)} .
\end{aligned}
$$


Closing this passage through Theorem 2.1, we turn our attention to its applications. So, our next concern is a mapping $f \rightarrow \int_{\sigma(A) \times \sigma(B)} f d \mu_{X, Y}$, which represents a bounded linear functional on $L^{\infty}\left(\sigma(A) \times \sigma(B), d \mu_{X, Y}\right)$ because

$$
\left|\int_{\sigma(A) \times \sigma(B)} f d \mu_{X, Y}\right| \leq\|f\|_{\infty}\left|\mu_{X, Y}\right|\left(\mathbb{C}^{2}\right) \leq\|f\|_{\infty}\|X\|_{2}\|Y\|_{2} .
$$

An equivalent interpretation of (6) will say that for every bounded, Borel measurable function $f$ defined on $\sigma(A) \times \sigma(B)$, and for every $Y \in \mathcal{C}_{2}(\mathcal{H})$, the mapping $X \rightarrow \int_{\sigma(A) \times \sigma(B)} f d \mu_{X, Y}$ is a bounded linear functional on $\mathcal{C}_{2}(\mathcal{H})$. Therefore, there exists a bounded linear operator on $\mathcal{C}_{2}(\mathcal{H})$, which will be denoted by $f(\mathcal{A}, \mathcal{B})$, such that

$$
\operatorname{tr}\left((f(\mathcal{A}, \mathcal{B}) X) Y^{*}\right)=\int_{\sigma(A)} \int_{\sigma(B)} f(z, w) \operatorname{tr}\left(E(d z) X F(d w) Y^{*}\right) .
$$

In the sequel we will preferably refer to $f(\mathcal{A}, \mathcal{B})$ as a (essentially bounded) $\mathfrak{C}_{2}(\mathcal{H})$ multiplier, rather than a $\mathfrak{C}_{2}(\mathcal{H})$ operator or transformer. More descriptive notation $\int_{\sigma(A)} \int_{\sigma(B)} f(z, w) \operatorname{tr}\left(E(d z) X F(d w) Y^{*}\right)$, which slightly differs from still cannonical $\int_{\sigma(A)} \int_{\sigma(B)} f(z, w) d \operatorname{tr}\left(E(z) X F(w) Y^{*}\right)$, will be used here instead of possibly rigid $\int_{\sigma(A) \times \sigma(B)} f d \mu_{X, Y}$ (in fact, this was already done in (7)). If $X=Y$, then we denote the same integral by $\int_{\sigma(A)} \int_{\sigma(B)} f(z, w)\|E(d z) X F(d w)\|_{2}^{2}$. For given $X \in \mathcal{C}_{2}(\mathcal{H})$ we use double operator integral $\int_{\sigma(A)} \int_{\sigma(B)} f(z, w) E(d z) X F(d w)$ to denote $f(\mathcal{A}, \mathcal{B}) X=f(\mathcal{A}, \mathcal{B})(X)$.

For the norm of such a $\mathcal{C}_{2}(\mathcal{H})$ operator we have $\|f(\mathcal{A}, \mathcal{B})\| \leq\|f\|_{\infty}$ by $(6)$, but a more precise observation will easily show that it is in fact an equality.

It is just a matter of straightforward calculation to show the validity of the following formulas in this functional calculus:

(i) $\mathcal{A} X=A X$ and $\mathcal{B} X=X B$.

(ii) $(\alpha f+\beta g)(\mathcal{A}, \mathcal{B}) X=\alpha f(\mathcal{A}, \mathcal{B}) X+\beta g(\mathcal{A}, \mathcal{B}) X$,

(iii) $(f g)(\mathcal{A}, \mathcal{B}) X=f(\mathcal{A}, \mathcal{B})(g(\mathcal{A}, \mathcal{B}) X)$,

(iv) $\sigma(f(\mathcal{A}, \mathcal{B}))$ is the essential range of $\sigma(A) \times \sigma(B)$ by $f$, measured by $\mu \times \nu$, for any positive measures $\mu$ and $\nu$ mutually absolutely continuous with respect to $E$ and $F$ respectively.

(v) $f(\mathcal{A}, \mathcal{B})^{*}=\bar{f}(\mathcal{A}, \mathcal{B})$.

From (iii) and (v) follows that $f(A, B)$ is a normal operator on $\mathfrak{C}_{2}(\mathcal{H})$, and also that

(vi) $\operatorname{tr}\left(f(\mathcal{A}, \mathcal{B}) X(g(\mathcal{A}, \mathcal{B}) Y)^{*}\right)=\operatorname{tr}\left(((f \bar{g})(\mathcal{A}, \mathcal{B}) X) Y^{*}\right)$.

Normality of $f(\mathcal{A}, \mathcal{B})$ could also be seen as follows:

Theorem 2.2 (Fuglede-Putnam for essentially bounded multipliers). For every essentially bounded Borel function $f$ and for all $X \in \mathfrak{C}_{2}(\mathcal{H})$,

$$
\|f(\mathcal{A}, \mathcal{B}) X\|_{2}=\left\|f(\mathcal{A}, \mathcal{B})^{*} X\right\|_{2} .
$$

The assertion of this theorem remains true for all bounded $X$ with finite left or right hand side in (8) if $f$ is an analytic multiplier. This is a central result in [J93]. For the related aspects of the standard derivation multiplier $X \rightarrow A X-X B$ see [Fur] and [N], and for other generalization of (8) see [W83], [W78] and [W81]. 
Here, we would also like to point out another important special case of (iii): if $f$ is a Lipschitz function on $\sigma(A) \cup \sigma(B)$, then

$$
f(A) X-X f(B)=\int_{\sigma(A)} \int_{\sigma(B)} \frac{f(z)-f(w)}{z-w} E(d z)(A X-X B) F(d w) .
$$

A restricting aspect of this formula is that $X$ has to be in $\mathfrak{C}_{2}(\mathcal{H})$, which obviously does not include the identity operator $I$ on any infinite dimensional Hilbert space $\mathcal{H}$. So this essentially bounded functional calculus is not fully applicable (at least not in this form) to perturbations or $A-B$ nor the generalized normal derivations $A X-X B$ on $\mathfrak{B}(\mathcal{H})$. Nevertheless, we will show that this formula holds as well for all $X$ in $\mathfrak{B}(\mathcal{H})$ having a Hilbert-Schmidt class derivation $A X-X B$; this is exactly the content of our next section.

\section{Derivation Formula}

Theorem 3.1 (Derivation formula). Let $A$ and $B$ be normal bounded operators such that $A X-X B \in \mathfrak{C}_{2}(\mathcal{H})$ for some $X \in \mathfrak{B}(\mathcal{H})$. Then for every Lipschitz function $f$ defined on $\sigma(A) \cup \sigma(B)$ we also have $f(A) X-X f(B) \in \mathfrak{C}_{2}(\mathcal{H})$. Furthermore, (1) also holds, i.e.,

$$
f(A) X-X f(B)=\check{f}(\mathcal{A}, \mathcal{B})(A X-X B),
$$

where

$$
\check{f}(z, w)= \begin{cases}(f(z)-f(w))(z-w)^{-1}, & \text { for } z \neq w \\ 0, & \text { for } z=w .\end{cases}
$$

Remark. The value of $\check{f}$ on a diagonal set $\{(z, z): z \in \sigma(A) \cup \sigma(B)\}$ in this integral formula is irrelevant just because $\mu_{A X-X B}$ vanishes there. For differentiable $f$ it is often more convenient to (re)define $\check{f}(z, z)=f^{\prime}(z)$ for all $z \in \sigma(A) \cap \sigma(B)$.

Proof. First, we will 'split' this formula on their local ingredients (and then, of course, recover it from scratch). Reducing the spectrum outside the diagonal set by the localization technique, we will be able to apply essentially bounded functional calculus because $X$ 'locally' belongs to the Hilbert-Schmidt class. Having both sides in formula (10) vanishing along the diagonal set, a controlled limit process will allow us to 'extend' this calculus to the missing diagonal set also.

So, let $\delta_{k, l}^{n}=\left[\frac{k}{n}, \frac{k+1}{n}\right) \times\left[\frac{l}{n}, \frac{l+1}{n}\right)$ with $\lambda_{k, l}$ as its center and let $\left\{\delta_{i}\right\}_{i=1}^{I}$ be a reindexation of (finite) covering $\left\{\delta_{k, l}^{n}\right\}$ for the set $\sigma(A) \cup \sigma(B)$. For $i, j \in I$ let us denote $E_{i}=E\left(\delta_{i}\right)$ and $F_{j}=F\left(\delta_{j}\right)$, as well as $A_{i}=A \uparrow_{E_{i}(\mathcal{H})}, B_{j}=B \uparrow_{F_{j}(\mathcal{H})}$ and $X_{i, j}=E_{i} X \uparrow_{F_{j}(\mathcal{H})}$. By a well known spectral theory fact we have $\sigma\left(A_{i}\right) \subset \bar{\delta}_{i}$ and $\sigma\left(B_{j}\right) \subset \bar{\delta}_{j}$. For $i=\left(k_{1}, l_{1}\right), j=\left(k_{2}, l_{2}\right)$ we define $\|i-j\|:=\max \left\{\left|k_{1}-k_{2}\right|,\left|l_{1}-l_{2}\right|\right\}$. So, if $\|i-j\|>1$, then $\bar{\delta}_{i} \cap \bar{\delta}_{j}=\emptyset$. According to the inversion formula for holomorphic calculus (see $[\mathrm{DK}],[\mathrm{R} 56]$ or $[\mathrm{Fi}]$ ), it follows that

$$
X_{i, j}=\oint_{\Gamma_{A_{i}}} \oint_{\Gamma_{B_{j}}}(z-w)^{-1}\left(A_{i}-z\right)^{-1}\left(A_{i} X_{i, j} F_{j}-E_{i} X_{i, j} B_{j}\right)\left(B_{j}-w\right)^{-1} d z d w .
$$

But $A_{i} X_{i, j} F_{j}-E_{i} X_{i, j} B_{j}=E_{i}(A X-X B) \uparrow_{F_{j}(\mathcal{H})}$, and therefore, it is a HilbertSchmidt class operator from $F_{j}(\mathcal{H})$ to $E_{i}(\mathcal{H})$. By $(12)$ the same thing happens to 
$X_{i, j}$ itself, which means that $E_{i} X F_{j} \in \mathfrak{C}_{2}(\mathcal{H})$. So according to essentially bounded functional calculus we have

$$
\check{f}(\mathcal{A}, \mathcal{B})\left(A E_{i} X F_{j}-E_{i} X F_{j} B\right)=f(A) E_{i} X F_{j}-E_{i} X F_{j} f(B),
$$

together with the fact that

$$
\left\|f(A) E_{i} X F_{j}-E_{i} X F_{j} f(B)\right\|_{2} \leq L\left\|A E_{i} X F_{j}-E_{i} X F_{j} B\right\|_{2} .
$$

Finally, we have to somehow include all those $\|i-j\| \leq 1$ in the same formula. To do this, we will consider $\mathcal{C}_{2}(\mathcal{H})$ projectors $\mathcal{Q}_{n}$ defined by $\mathcal{Q}_{n}(Z)=\sum_{\|i-j\| \leq 1} E_{i}^{2^{n}} Z F_{j}^{2^{n}}$, for all $Z \in \mathfrak{C}_{2}(\mathcal{H})$. By Theorem 2.8.6 from [BS80] we conclude that the monotonically decreasing projector family $\left\{\mathcal{Q}_{n}\right\}_{n \geq 1}$ is strongly convergent on $\mathcal{C}_{2}(\mathcal{H})$, and therefore, there exists the following $\mathcal{C}_{2}-\lim _{n \rightarrow \infty} \mathcal{Q}_{n}(A X-X B)$. To determine this limit, for fixed $a$ and $b$ in $\{-1,0,1\}$, let $\lambda_{i^{\prime}}=\lambda_{i}+2^{-n}(a+\imath b)$ denote the center of the square $\delta_{i^{\prime}}$ neighboring $\delta_{i}$. In each of those cases we have

$$
\begin{aligned}
& \left|\left\langle\sum_{i=1}^{I} E_{i}^{2^{n}}(A X-X B) F_{i^{\prime}}^{2^{n}} g, h\right\rangle+2^{-n}(a+\imath b)\langle X g, h\rangle\right| \\
& =\left|\sum_{i=1}^{I}\left(\left\langle E_{i}^{2^{n}}\left(A-\lambda_{i}\right) X F_{i^{\prime}}^{2^{n}} g, h\right\rangle-\left\langle E_{i}^{2^{n}} X\left(B-\lambda_{i^{\prime}}\right) F_{i^{\prime}}^{2^{n}} g, h\right\rangle\right)\right| \\
& \quad \leq \sum_{i=1}^{I}\left(\left\|X F_{i^{\prime}}^{2^{n}} g\right\|\left\|\left(A-\lambda_{i}\right)^{*} E_{i}^{2^{n}} h\right\|+\left\|\left(B-\lambda_{i^{\prime}}\right) F_{i^{\prime}}^{2^{n}} g\right\|\left\|X^{*} E_{i}^{2^{n}} h\right\|\right) \\
& \leq 2 \sum_{i=1}^{I} 2^{-n}\|X\|\left\|F_{i^{\prime}}^{2^{n}} g\right\|\left\|E_{i}^{2^{n}} h\right\| \leq 2^{1-n}\|X\|\|g\|\|h\|,
\end{aligned}
$$

and so

$$
\left\|\sum_{i=1}^{I} E_{i}^{2^{n}}(A X-X B) F_{i^{\prime}}^{2^{n}}\right\| \leq 2^{-n}(2+|a+\imath b|)\|X\| .
$$

Splitting $\left\langle\mathcal{Q}_{n}(A X-X B) g, h\right\rangle$ into nine such sums, we get

$$
\left\|\mathcal{Q}_{n}(A X-X B)\right\| \leq 2^{-n}(18+1 \cdot 0+4 \cdot 1+4 \sqrt{2})\|X\| \longrightarrow 0
$$

as $n \longrightarrow \infty$, and quite similarly $\lim _{n \rightarrow \infty}\left\|\mathcal{Q}_{n}(f(A) X-X f(B))\right\|=0$. By virtue of (14) we have

$$
\begin{aligned}
& \left\|\left(I-\mathcal{Q}_{n}\right)(f(A) X-X f(B))\right\|_{2}^{2}=\sum_{\|i-j\| \geq 1}\left\|E_{i}(f(A) X-X f(B)) F_{j}\right\|_{2}^{2} \\
& \quad \leq \quad L^{2} \sum_{\|i-j\| \geq 1}\left\|E_{i}(A X-X B) F_{j}\right\|_{2}^{2} \leq L^{2}\|A X-X B\|_{2}^{2},
\end{aligned}
$$

and then, according to the lower semicontinuity of $\|\cdot\|_{2}$, it follows that

$$
\begin{aligned}
& \|f(A) X-X f(B)\|_{2}=\left\|w-\lim _{n \longrightarrow \infty}\left(I-\mathcal{Q}_{n}\right)(f(A) X-X f(B))\right\|_{2} \\
& \quad \leq \liminf _{n \longrightarrow \infty}\left\|\left(I-\mathcal{Q}_{n}\right)(f(A) X-X f(B))\right\|_{2} \leq L\|A X-X B\|_{2} .
\end{aligned}
$$

Therefore, the same Theorem 2.8.6 from [BS80] is applicable to $f(A) X-X f(B)$, and so $\mathcal{C}_{2^{-}} \lim _{n \longrightarrow \infty} \mathcal{Q}_{n}(f(A) X-X f(B))=0$, together with the already shown 
fact that $\mathcal{C}_{2}-\lim _{n \rightarrow \infty} \mathcal{Q}_{n}(A X-X B)=0$. Now, a straightforward calculation gives:

$$
\begin{aligned}
& f(A) X-X f(B)-\check{f}(\mathcal{A}, \mathcal{B})(A X-X B) \\
& \quad=\sum_{\|i-j\| \geq 1}\left(E_{i}(f(A) X-X f(B)) F_{j}-\check{f}(\mathcal{A}, \mathcal{B}) E_{i}(A X-X B) F_{j}\right) \\
& \quad+\quad \mathcal{Q}_{n}(f(A) X-X f(B))-\check{f}(\mathcal{A}, \mathcal{B}) \mathcal{Q}_{n}(A X-X B) \\
& \quad=\quad \mathcal{Q}_{n}(f(A) X-X f(B))-\check{f}(\mathcal{A}, \mathcal{B}) \mathcal{Q}_{n}(A X-X B),
\end{aligned}
$$

as follows from (13). Having that the last two terms tend to zero in the norm of $\mathfrak{C}_{2}(\mathcal{H})\left(\check{f}(\mathcal{A}, \mathcal{B})\right.$ is a bounded $\mathfrak{C}_{2}(\mathcal{H})$ operator $)$, we finally get $\check{f}(\mathcal{A}, \mathcal{B})(A X-X B)=$ $f(A) X-X f(B)$, as desired.

We may also see that if we have two Lipschitz functions $f$ and $g$, then an immediate consequence will be

$$
\begin{aligned}
& \operatorname{tr}(f(A) X-X f(B))(g(A) Y-Y g(B))^{*} \\
& \quad=\operatorname{tr}\left(\check{f}(\mathcal{A}, \mathcal{B})(A X-X B)(\check{g}(\mathcal{A}, \mathcal{B})(A Y-Y B))^{*}\right),
\end{aligned}
$$

which, according to the basic properties of double operator integrals, gives

Corollary 3.1. Under conditions of Theorem 1.1, for all Lipschitz functions $f$ and $g$ defined on $\sigma(A) \cup \sigma(B)$, the following holds:

$$
\begin{aligned}
\operatorname{tr} & (f(A) X-X f(B))(g(A) Y-Y g(B))^{*} \\
\quad= & \int_{\sigma(A)} \int_{\sigma(B)} \check{f}(z, w) \overline{\check{g}(z, w)} \operatorname{tr}\left(E(d z)(A X-X B) F(d w)(A Y-Y B)^{*}\right) .
\end{aligned}
$$

Consequently,

$$
\operatorname{tr}(f(A) X-X f(B))(g(A) X-X g(B))^{*} \in\|A X-X B\|_{2}^{2} \overline{\operatorname{Co}}(\check{f} \check{g})(\sigma(A), \sigma(B)),
$$

where $\overline{\mathrm{Co}}$ stands for the closed convex hull of a given set.

For $f=g$, an immediate consequence of this corollary is Theorem 1.1 of Kittaneh, which, in turn, gives in its special case $f(z)=\bar{z}$ that

$$
\left\|A^{*} X-X B^{*}\right\|_{2}=\|A X-X B\|_{2} .
$$

This is a considerable straightening of the classical Fuglede-Putnam theorem (see [Fug], [P], [R58] and [Be]), because $A X-X B=0$ implies $A^{*} X-X B^{*}=0$ by (17).

The following theorem is a straightforward consequence of the above derivation formula and generalizes some inequalities of Muir and Wong for covariance matrices, given in $[\mathrm{Mu}]$ and $[\mathrm{Wo}]$.

Theorem 3.2. Let some self-adjoint bounded operators $A$ and $B$ be such that $A X-$ $X B \in \mathfrak{C}_{2}(\mathcal{H})$ for some $X \in \mathfrak{B}(\mathcal{H})$. If $f$ and $g$ are monotonically increasing Lipshitz functions defined on $\sigma(A) \cup \sigma(B)$, then

$$
\operatorname{tr}(f(A) X-X f(B))(g(A) X-X g(B))^{*} \geq 0 .
$$

In particular, if $A$ and $B$ are invertible, then for all real $\alpha$ and $\beta$ the following holds:

$$
(\alpha+1)(\beta+1) \operatorname{tr}\left(A|A|^{\alpha} X-X B|B|^{\alpha}\right)\left(A|A|^{\beta} X-X B|B|^{\beta}\right)^{*} \geq 0 .
$$

If $X=I, A \neq B$, and $\alpha, \beta \neq-1$, the last inequality is strict. 
We proceed with related norm inequalities for operator monotone functions. Some of them could also be derived by considering commuting (normal) multiplication operators $\mathcal{A} X=A X$ and $\mathcal{B} X=X B$ on $\mathcal{C}_{2}(\mathcal{H})$, but here we show how they can naturally be derived in the framework of double operator integrals.

Theorem 3.3 (Means and related inequalities). Let $A$ and $B$ be bounded, positive operators and let $f$ be a non-negative operator monotone function defined on $[0,+\infty)$. Then for all Hilbert-Schmidt class operators $X$

$$
\frac{\|f(A) X+X f(B)\|_{2}}{2\|X\|_{2}} \leq f\left(\frac{\|A X+X B\|_{2}}{2\|X\|_{2}}\right),
$$

and

$$
\|f(A) X-X f(B)\|_{2} \leq\|X\|_{2} f\left(\frac{\|A X-X B\|_{2}}{\|X\|_{2}}\right),
$$

and particularly,

$$
\left(\frac{\left\|A^{\alpha} X+X B^{\alpha}\right\|_{2}}{2\|X\|_{2}}\right)^{\frac{1}{\alpha}} \leq\left(\frac{\left\|A^{\beta} X+X B^{\beta}\right\|_{2}}{2\|X\|_{2}}\right)^{\frac{1}{\beta}},
$$

for all $0<\alpha \leq \beta$.

Proof. Without loss of generality we may suppose that $\|X\|_{2}=1$. If $A$ or $B$ is invertible, then essentially bounded calculus is fully applicable, and therefore a straightforward computation shows that

$$
\frac{\|f(A) X+X f(B)\|_{2}^{2}}{4}=\iint_{\sigma(A) \times \sigma(B)}\left(\frac{f(z)+f(w)}{2}\right)^{2} d \mu_{X}(z, w),
$$

where $d \mu_{X}(z, w)=\|E(d z) X F(d w)\|_{2}^{2}$. Every such operator monotone function is concave (see [BSh], $[\mathrm{Kw}]$ or $[\mathrm{Do}]$ ), and therefore

$$
\begin{gathered}
\frac{\|f(A) X+X f(B)\|_{2}^{2}}{4} \leq \iint_{\sigma(A) \times \sigma(B)} f^{2}\left(\frac{z+w}{2}\right) d \mu_{X}(z, w) \\
=\iint_{\sigma(A) \times \sigma(B)} g\left(\left(\frac{z+w}{2}\right)^{2}\right) d \mu_{X}(z, w),
\end{gathered}
$$

for a function $g$ defined by $g(u)=f^{2}(\sqrt{u})$. According to [FKK], $g$ is also an operator monotone function, and therefore concave. Now, an application of Jensen's inequality to concave function $g$ and a probability measure $\mu_{X}$ (total mass $\mu_{X}\left([0,+\infty)^{2}\right)$ $=\|X\|_{2}^{2}=1$ ) gives

$$
\begin{aligned}
& \frac{\|f(A) X+X f(B)\|_{2}^{2}}{4} \\
& \quad \leq g\left(\iint_{\sigma(A) \times_{\sigma}(B)}\left(\frac{z+w}{2}\right)^{2} d \mu_{X}(z, w)\right)=f^{2}\left(\frac{\|A X+X B\|_{2}}{2}\right) .
\end{aligned}
$$

Therefore, (18) follows for invertible $A$ or $B$, in particular

$$
\frac{\|f(A+\epsilon) X+X f(B+\epsilon)\|_{2}}{2} \leq f\left(\frac{\|(A+\epsilon) X+X(B+\epsilon)\|_{2}}{2}\right),
$$


for all $\epsilon>0$. Another appeal to the lower semicontinuity of $\|\cdot\|_{2}$ gives

$$
\begin{aligned}
\frac{\|f(A) X+X f(B)\|_{2}}{2} & =\left\|s-\lim _{\epsilon \longrightarrow 0} \frac{f(A+\epsilon) X+X f(B+\epsilon)}{2}\right\|_{2} \\
& \leq \lim \inf _{\epsilon \longrightarrow 0} f\left(\frac{\|(A+\epsilon) X+X(B+\epsilon)\|_{2}}{2}\right) \\
& \leq \lim \inf _{\epsilon \longrightarrow 0} f\left(\frac{\|A X+X B\|_{2}}{2}+\epsilon\right) \\
& =f\left(\frac{\|A X+X B\|_{2}}{2}\right),
\end{aligned}
$$

due to the continuity of operator monotone function $f$.

Statement (19) could be proved similarly, taking into account the fact that $|g(z)-g(w)| \leq g(|z-w|)$ for $z, w>0$, as known from [A], for example.

To prove (20), we apply (18) to positively defined operators $A^{\beta}$ and $B^{\beta}$ and operator monotone function $t \longrightarrow t^{\frac{\alpha}{\beta}}$.

Inequalities (18) and (20) turn out to be equalities for $A=B=X=\langle\cdot, f\rangle, f$ a one-dimensional (orthogonal projection) operator for any (unit) vector $f \in \mathcal{H}$, and the same happens to be true in (19) for $A=X=\langle\cdot, f\rangle f$ and $B=0$. This shows that all constants appearing in the above inequalities are optimal; and each of those inequalities is (at least) as precise as it was for scalars.

For non-real $\alpha$ a change of constants is inevitable even for scalar multiples of an identity operator on finite-dimensional Hilbert spaces. With the help of double operator integrals we will show that some scalar inequalities still hold for their appropriate classes of operators.

Theorem 3.4. Let $A, B \geq 0, X \in \mathcal{C}_{2}(\mathcal{H})$ and let $\alpha$ be a complex number in the strip $0 \leq \Re \mathrm{e} \alpha<1$. Then

$$
\begin{gathered}
\left\|A^{\alpha} X-X B^{\alpha}\right\|_{2} \leq \frac{|\sin \pi \alpha|}{\sin (\pi \Re \mathrm{e} \alpha)}\|X\|_{2}^{1-\Re \mathrm{e} \alpha}\|A X-X B\|_{2}^{\Re \mathrm{e} \alpha}, \\
\left\|A^{\alpha} X+X B^{\alpha}\right\|_{2} \leq 2^{1-\Re \mathrm{e} \alpha} \frac{|\sin \pi \alpha|}{\sin (\pi \Re \mathrm{e} \alpha)}\|X\|_{2}^{1-\Re \mathrm{e} \alpha}\|A X+X B\|_{2}^{\Re \mathrm{e} \alpha},
\end{gathered}
$$

and also

$$
\begin{gathered}
\left\|A^{\alpha}-B^{\alpha}\right\| \leq \frac{|\sin \pi \alpha|}{\sin (\pi \Re \mathrm{e} \alpha)}\left\||A-B|^{\Re \mathrm{e} \alpha}\right\|, \\
\left\|A^{\alpha}+B^{\alpha}\right\| \leq 2^{1-\Re \mathrm{e} \alpha} \frac{|\sin \pi \alpha|}{\sin (\pi \Re \mathrm{e} \alpha)}\left\||A+B|^{\Re \mathrm{e} \alpha}\right\|,
\end{gathered}
$$

for all unitarily invariant norms $\|\cdot\| \cdot$

Proof. From integral representations $A^{\alpha}=\frac{\sin \pi \alpha}{\pi} \int_{0}^{\infty} A(A+t)^{-1} t^{\alpha-1} d t$ and $B^{\alpha}=$ $\frac{\sin \pi \alpha}{\pi} \int_{0}^{\infty} B(B+t)^{-1} t^{\alpha-1} d t$ we get

$$
\left\|A^{\alpha} X-X B^{\alpha}\right\|_{2} \leq \frac{|\sin \pi \alpha|}{\pi} \int_{0}^{\infty}\left\|A(A+t)^{-1} X-X B(B+t)^{-1}\right\|_{2} t^{\Re \mathrm{e} \alpha-1} d t .
$$


As $A \longrightarrow A(A+t)^{-1}$ is operator monotone for each $t>0$, it follows from (19) that for $\|X\|_{2}=1$,

$$
\begin{aligned}
& \left\|A^{\alpha} X-X B^{\alpha}\right\|_{2} \\
& \quad \leq \frac{|\sin \pi \alpha|}{\pi} \int_{0}^{\infty} \frac{\|A X-X B\|_{2}}{t+\|A X-X B\|_{2}} t^{\Re \mathrm{e} \alpha-1} d t=\frac{|\sin \pi \alpha|}{\sin (\pi \Re \mathrm{e} \alpha)}\|A X-X B\|_{2}^{\Re \mathrm{e} \alpha},
\end{aligned}
$$

from which (22) easily follows for $\|X\|_{2} \neq 1$ as well.

The proof for (23) only differs in an application of (18) instead of (19).

To prove (24) we note that for all Ky-Fan $k$-norms $\|\cdot\|_{k}, k=1,2, \ldots$, the following holds:

$$
\begin{aligned}
\| A^{\alpha} & -B^{\alpha}\left\|_{k} \leq \frac{|\sin \pi \alpha|}{\pi} \int_{0}^{\infty}\right\| A(A+t)^{-1}-B(B+t)^{-1} \|_{k} t^{\Re \mathrm{e} \alpha-1} d t \\
& \leq \frac{|\sin \pi \alpha|}{\pi} \int_{0}^{\infty}\left\|\frac{|A-B|}{t+|A-B|}\right\|_{k} t^{\Re \mathrm{e} \alpha-1} d t \\
& =\frac{|\sin \pi \alpha|}{\pi} \int_{0}^{\infty} \sum_{i-1}^{k} \frac{s_{i}(A-B)}{t+s_{i}(A-B)} t^{\Re \mathrm{e} \alpha-1} d t \\
& =\frac{|\sin \pi \alpha|}{\sin (\pi \Re \mathrm{e} \alpha)}\left\||A-B|^{\Re \mathrm{e} \alpha}\right\|_{k},
\end{aligned}
$$

where (26) follows by the theorem of Ando in [A], especially when applied to operator monotone function $A \longrightarrow A(A+t)^{-1}$ for all $t>0$. By the Ky-Fan dominance principle we conclude (24) for all unitarily invariant norms $\|\cdot\| \cdot \|$.

The proof for the last assertion of our theorem is quite similar to the previous one, and the only essential difference consists of an application of the inequality

$$
\left\|\frac{A(A+t)^{-1}+B(B+t)^{-1}}{2}\right\|_{k} \leq\left\|\frac{(A+B)}{2}\left(\frac{A+B}{2}+t\right)^{-1}\right\|_{k},
$$

which is, in turn, a straightforward consequence of the concavity (in the operator order $\leq$ ) for the operator monotone function $A \longrightarrow A(A+t)^{-1}$ for all $t>0$, as shown in $[\mathrm{Kw}]$, for example.

The last two theorems extend results of $[\mathrm{A}]$ and $[\mathrm{BKS}]$ to non-real $\alpha$, saving the original real case constants; moreover, they improve constants appearing in [Da] and $[\mathrm{Bo}]$ applications to $\mathfrak{C}_{2}(\mathcal{H})$. Inequality (20) has been recently generalized to all unitarily invariant norms in $[\mathrm{J}]$, but only under restricting condition $\beta \geq 2 \alpha$. We also recommend that the reader compare those constants with their analogous for self-adjoint derivations treated in [JK] and [J97], to realize that in both cases, our norm inequalities for operators are still inheriting (despite its substantial domain extension) the unchanged constants from their parallel scalar inequalities.

Finally, we conclude this paper believing that both (22) and (24) are just special consequences of the following:

\section{Hypothesis 3.1.}

$$
\sum_{i=1}^{k} s_{i}^{p}\left(A^{\alpha} X-X B^{\alpha}\right) \leq \frac{|\sin \pi \alpha|}{\sin (\pi \Re \mathrm{e} \alpha)} \sum_{i=1}^{k} s_{i}^{p(1-\Re \mathrm{e} \alpha)}(X) s_{i}^{p \Re \mathrm{e} \alpha}(A X-X B),
$$

for some $p \geq 1$ and for all $0 \leq \alpha \leq 1$ and $k=1,2, \ldots$. 


\section{REFERENCES}

[A] T. Ando, Comparison of norms $\|f(A)-f(B)\|$ and $\|f(|A-B|)\|$, Math. Z. 197 (1988), 403-408. MR 90a:47021

[BSh] J. Bendat and S. Sherman, Monotone and Convex Operator Functions, Trans. Amer. Math. Soc., 79 (1955), 58-71. MR 18:588b

[Be] S.K. Berberian, Extensions of the theorem of Fuglede and Putnam, Proc. Amer. Math. Soc. 71 (1978), 113-114. MR 58:7176

[BKS] M.Sh. Birman, L.S. Koplienko and M.Z. Solomyak, Estimates for the spectrum of the difference between fractional powers of two self-adjoint operators, (Russian), Izv. Vissh. Uchebn. Zav. Mat. 1975, 3-10. MR 52:6458

[BS66] M.Sh. Birman, M.Z. Solomyak, Double operator Stiltjes integrals, (Russian), Problemi matematicheskoj fiziki, vyp 1, Izd. LGU, 33-76, 1966.

[BS68] M.Sh. Birman, M.Z. Solomyak, Double operator Stiltjes integrals II, (Russian), Problemi matematicheskoj fiziki, vyp 3, Izd. LGU, 81-88, 1968.

[BS69] M.Sh. Birman, M.Z. Solomyak, Double operator Stiltjes integrals III, (Russian), Problemi matematicheskoj fiziki, vyp 3, Izd. LGU, 27-53, 1969.

[BS80] M.Sh. Birman and M.Z. Solomyak, Spektral'naya teoriya samosopryazhenni'h operatorov $v$ Gil'bertovom prostranstve, (Russian), Izd-vo Leningr. Univ, 1980.

[Bo] K.N. Boyadzhiev, Some inequalities for generalized commutators, Publ. RIMS, Kyoto Univ. 26 (1990), 521-527. MR 93c: 47009

$[\mathrm{CF}]$ I. Colojoara and C. Foias, Theory of generalized spectral operators, Gordon and Breach, New York 1968. MR 52:15085

[DK] Yu.L. Daleckij, M.G. Krein: Ustoichivost reshenij differenciyal'nih uravnenij v Banahovom prostranstve, Nauka, Moskva 1972.

[Da] E.B. Davies, Lipschitz continuity of functions of operators in the Schatten classes, J. London Math. Soc. (2) 37 (1988), 148-157. MR 89c:47009

[Do] J. Donoghue, Monotone matrix functions and analytic continuation, Berlin, Heidelberg, New York, Springer 1974.

[Fa] Yu.B. Farfarovskaya, Example of Lipschitz function of self-adjoint operators thet gives a nonnuclear increment under a nuclear perturbation, J. Soviet Math. 4 (1975), 426-433.

[Fi] L. Fialkow, $A$ note on the operator $X \rightarrow A X-X B$, Trans. Amer. Math. Soc. (9) 243 (1978), 147-168. MR 80c:47017

[Fug] B. Fuglede, A commutativity theorem for normal operators, Proc. Nat. Acad Sci. U.S.A. 36 (1950), 35-40. MR 11:371c

[FKK] J.I. Fujii, F. Kubo and K. Kubo, A parametrization between operator means, Math. Japonica (2) 33 (1988), 201-208. MR 89i:47027

[Fur] T. Furuta, A Hilbert-Schmidt norm inequalitiy associated with the Fuglede -Putmam theorem, Ark. Mat. (Basel) 20 (1982), 157-163. MR 83g:47021

[GGK] I. Gohberg, S. Goldberg and M.A. Kaashoek, Classes of linear operators, Operator Theory Vol. 49, Birkhauser Verlag, Basel 1990. MR 93d:47002

[GK] I.C. Gohberg and M.G. Krein, Introduction to the theory of linear nonselfadjoint operators, Transl. Math. Monographs, vol. 18, Amer. Math. Soc. Providence, R.I. 1969. MR 39:7447

[HJ] R. Horn and C. Johnson, Topics in Matrix Analysis, Cambridge University Press, Cambridge, 1990. MR 95c:15001; MR 92e:15003

[JK] D. Jocić and F. Kittaneh, Some perturbation inequalities for self-adjoint operators, J. Operator Theory, 31 (1994), 3-10. MR 96b:47017

[J93] D.R. Jocić, A Hilbert-Schmidt norm equality associated with the Fuglede-PutnamRosenblum's type theorem for generalized multipliers, J. Operator Theory 30 (1993), 31-40. MR 96h:47028

[J97] D.R. Jocić, Norm inequalities for self-adjoint derivartions, J. Functional Analysis 145 (1997) 24-34. MR 98b:47031

[J] D.R. Jocić, Cauchy-Schwarz and means inequalities for elementary operators into norm ideals, Proc. Amer. Math. Soc. 126 (1998), 2705-2711. CMP 97:13

[Ki] F. Kittaneh, On Lipschitz functions of normal operators, Proc. Amer. Math. Soc. (3) 94 (1985) 416-418. MR 87b:47021

[Kw] M.K. Kwong, Some results on monotone matrix functions, Linear Algebra Appl. 118 (1989), 129-153. MR 90e:47016 
$[\mathrm{Mu}] \quad \mathrm{W} . \mathrm{W}$. Muir, Inequalities concerning the inverse of positive definite matrices, Proc. Edinbourgh Math. Soc. (2) 19 (1974/75), 109-113. MR 53:5633

[N] R. Nakamoto, A characterization of normal operators using the Hilbert-Schmidt class, Proc. Amer. Math. Soc. (2) 79 (1980) 313-314. MR 81b:47032

[P] C.R. Putnam, Commutation properties of Hilbert space operators and related topics, Berlin-Heidelberg-New York, 1967. MR 36:707

[R56] M. Rosenblum, On the operator equation $B X-X A=Q$, Duke Math. J. 23 (1956), 263-270. MR 18:54d

[R58] M. Rosenblum, On a theorem of Fuglede and Putnam, J. London Math. Soc. 33 (1958), 376-377. MR 20:6037

[Sh] V. Shulman, Some remarks on the Fuglede-Weiss theorem, Bull. London Math. Soc. 28 (1996), 385-392. MR 97c:47021

[Si] B. Simon, Trace Ideals and their applications, Cambridge University Press, 1979. MR 80k: 47048

[Vo] D. Voiculescu, Some results on norm ideal perturbation of Hilbert space operators, J. Operator Theory 2 (1979), 3-37. MR 80m:47012

[W78] G. Weiss, The Fuglede commutativity theorem modulo the Hilbert-Schmidt class and generating functions for matrix operators.I, Trans. Amer. Math. Soc. 246 (1978), 193209. MR 81b:47033

[W81] G. Weiss, The Fuglede commutativity theorem modulo the Hilbert-Schmidt class and generating functions for matrix operators. II, J. Operator Theory 5 (1981), 3-16. MR 83g: 47041

[W83] G. Weiss, An extension of the Fuglede commutativity theorem modulo the HilbertSchmidt class to the operators of the form $\sum M_{n} X N_{n}$, Trans. Amer. Math. Soc. 278 (1983), 1-20. MR 84e:47026

[Wo] C.S. Wong, Matrix derivative and its applications in statistics, J. Math. Phych., 22 (1980), 70-80. MR 81m:62105

University of Belgrade, Faculty of Mathematics, Studentski trg 16, P. O. Box 550, 11000 Belgrade, Yugoslavia

E-mail address: jocic@matf.bg.ac.yu 